\title{
A Novel Cooperative Network Using Down-link Non- orthogonal Multiple Access Scheme
}

\section{Syed Agha Hassnain Mohsan ( $\nabla$ Hassnainagha@zju.edu.cn )}

Zhejiang University https://orcid.org/0000-0002-5810-4983

\section{Moqbel Ali Mohammed Hamood}

Jordan University of Science and Technology

\section{Syed Muhammad Tayyab Shah}

COMSATS University Islamabad

\section{Alireza Mazinani}

Beihang University

\section{Research Article}

Keywords: downlink cooperative non-orthogonal multiple access (DC-NOMA), Scheme, Cooperative Network, Novel, NOMA schemes

Posted Date: November 30th, 2021

DOI: https://doi.org/10.21203/rs.3.rs-1059857/v1

License: (c) (i) This work is licensed under a Creative Commons Attribution 4.0 International License. Read Full License 


\title{
A Novel Cooperative Network Using Down-link Non-orthogonal Multiple Access Scheme
}

\author{
Syed Agha Hassnain Mohsan ${ }^{1}$, Moqbel Ali Mohammed Hamood ${ }^{2}$, \\ Syed Muhammad Tayyab Shah ${ }^{3}$, and Alireza Mazinani ${ }^{4}$ \\ ${ }^{1}$ Zheda Road 1, Zhoushan, Zhejiang 316021, China \\ ${ }^{2}$ Department of Electrical Engineering, Jordan University of Science and Technology, Irbid, Jordan \\ ${ }^{3}$ Department of Electrical Engineering, COMSATS University, Islamabad, Pakistan \\ ${ }^{4}$ School of Electronic and Information Engineering, Beihang University, Beijing, China
}

\begin{abstract}
A novel downlink cooperative non-orthogonal multiple access (DC-NOMA) scheme is proposed in this paper to achieve higher performance in the spectral efficiency compared to the classical NOMA schemes. The communication system consists of one base station and two users (e.g., strong user and weak user). In down link phase, the base station transmits a superimposed signal to both users, and in the cooperative phase, the weak user sends its decoded message to the strong user. The main idea is how the weak user can help the strong user to improve the performance of both users. This occurs by enabling the weak user to perform a cooperative transmission with the strong user during the cooperative phase. The outage probability, outage throughput, and diversity order are derived and analyzed. Numerical results are provided to show that the spectral efficiency gain achieved through our proposed scheme is better than the conventional cooperative NOMA schemes.
\end{abstract}

\section{INTRODUCTION}

The increase in the demands of the expected new services and data traffic, such as cloud-based architectural applications and among such, is the main challenge for the $5 \mathrm{G}$ wireless network. Thus, there are challenging requirements to achieve these services, such as high massive connectivity, much higher data rates (100-1000 times faster than current 4G technology), and low latency. As a result, these requirements cannot be achieved by using OMA [1-3]. Non-orthogonal multiple access (NOMA) is proposed to address the drawbacks of OMA scheme. Serving multiple users at the same time, frequency, code and space domain, but with different power levels, at the expense of minimal inter-user interference (IUI) is the main concept of NOMA scheme $[4,5]$. NOMA Scheme intends to share domains among users via superposition and consequently it requires to employ multiple user detection (MUD) strategy to separate interfered users sharing the same degree of freedom, as illustrated in Fig. 1 [6]. As result, interesting multiple access technique for the fifth generation (5G) network, can be obtained by NOMA scheme [7,8]. Moreover, NOMA techniques are classified into two domains, i.e. the power and code domains. Power domain NOMA is more interesting technique regarding to its implementation in $4 \mathrm{G}$ techniques. In addition, Serving and supporting multiple users with some degree of freedom (DoF) can be satisfied by using power domain NOMA, in which it performs successive interference cancellation (SIC) at receivers with better channel condition. In practice, multiplexing technique is achieved by using superposition coding at the transmitter and SIC at the receiver [10], [11]. On the other hand, the code domain NOMA uses user-specific spreading sequence which are either non-orthogonal cross-correlated sequences of low correlation coefficient or sparse sequences. This scheme is further divided into different classes, such as low-density spreading-based OFDM (LDS-OFDM), low-density spreading CDMA (LDS-CDMA), and sparse code multiple access (SCMA) $[12,13]$. Generally, there are three NOMA transmission scenarios viz. downlink NOMA transmission scenario, cooperative NOMA transmission scenario and uplink NOMA transmission scenario. In this paper, we take a consideration of the downlink NOMA transmission scenario as well as cooperative NOMA transmission scenario.

In [7], the authors proposed power domain user multiplexing technique at base-station (BS) based on the discrete Fourier transform (DFT)-spread OFDM or the orthogonal frequency division multiple access (OFDMA). In addition, they studied the successive interference cancellation (SIC)-based signal reception at receivers for robust multiple access. The overall system throughput proposed in [14] to show the superiority of NOMA over OMA schemes. These benefits include the following; higher spectral efficiency, fairness, and higher connection density The performance 


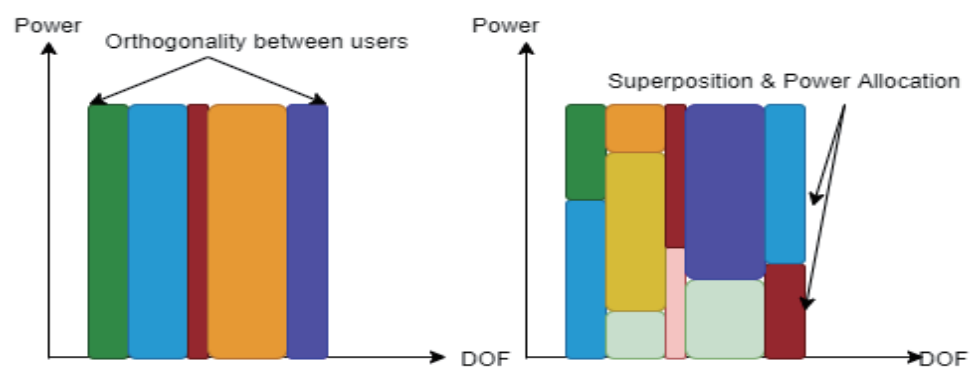

Figure 1: Comparison between NOMA and OMA in the power domain multiplexing [9].

of deployed users with NOMA scheme is investigated in [15]. In addition, for two users, they discussed the closed form expression of ergodic sum rate and outage probability. In contrast, the fairness's concept in downlink NOMA scheme with a known channel state information (CSI) feedback in-term of a power allocation was studied in [16]. In [17], the authors proposed a cooperative diversity technique that considers a suitable scheme to combat the multipath propagation fading in wireless channel. The space diversity between cooperating terminals will be exploited by cooperative diversity techniques to relaying signals from one terminal to another. In [18], they studied the cooperative relay to improve the physical layer security in the wireless medium. They exploited several advantages in cooperative relay such as provision of reduced access distance and distributed diversity. In [19], and [20], the authors proposed a cooperative relay, which is one of the important techniques to improve the link reliability coupled with an increase in the range of the communication system.

The authors in [21] proposed a cooperative NOMA (CNOMA) scheme which helps the weak user to increase its reception reliability by exploiting the prior information about the messages of other users obtained at the strong user. Particularly, in the NOMA scheme, the strong user acts as forward and decode (FD) relay to deliver the message to the weak user. In [22, 23], fullduplex relaying network, multiple-antenna relaying networks were studied which maybe considered as an extension of the (FD) relay in NOMA. In addition, the transmit antennas at the relay can increase the signal to-noise ratio (SNR), whereas the receive antennas at the mobile users adopt the Maximum Ratio Combining (MRC). As a result, it is noted that the spectral efficiency at the aforementioned NOMA schemes is reduced owing to the duplication in the transmission during the cooperative phase.

Recently, in $[24,25]$, non-orthogonal relay in CNOMA scheme was proposed to achieve higher spectral efficiency,however, this increment came at the expense of the signal reception reliability of the weak user. Noting that, in [26] the authors proposed a hybrid downlink-uplink CNOMA (HDUCNOMA) scheme with an aim to improve the spectral efficiency. In this scheme, the strong user can simultaneously perform uplink transmission and cooperative transmission during the cooperative phase. Nevertheless, the signal reception reliability has been slightly decreased in [26] scheme.

In this paper, we propose a novel downlink cooperative NOMA (DC-NOMA) scheme and investigate its performance. The given scheme performs its work in two stages, i.e., (1) First, the base-station transmits superimposed signal to the deployed users in a given geographic area. (2) the weak user, in the second step, transmits its decoded signal to the strong user. By comparison to other similar schemes, our scheme allocates more power to the weak user. This in turns promises an improved SINR for the weak user and consequently an improved channel capacity. Moreover, we also achieve higher SINR for the strong user with improved channel capacity. This implies, our proposed scheme provides better system performance through improved SINR at both strong and weak user.

This paper is organized as follows: Section 1 discusses the introduction of the paper including the motivation and contribution. The main contribution and organization of the paper are also summarized. In Section 2, the system model is presented which includes the introduction of the proposed scheme, the analysis of system performance for the DC-NOMA scheme with exponential fading channel. This section also includes the derivation of the outage probability for the proposed scheme, the diversity order and the throughput based on the outage probabilities to evaluate the performance of our system. In Section 3, The numerical results of our work is provided to verify the analytical results and to illustrate the effectiveness of our proposed scheme. In connection to this, in this section, we also presented the performances of the throughput and the diversity order 
by using the numerical results of the outage probability. In section 4, the summary and conclusion of the paper are discussed and the future research work and way-forward are given.

\section{SYSTEM MODEL}

A communication system model shown in Fig. 2 consists of a downlink transmission with one base station and two users.

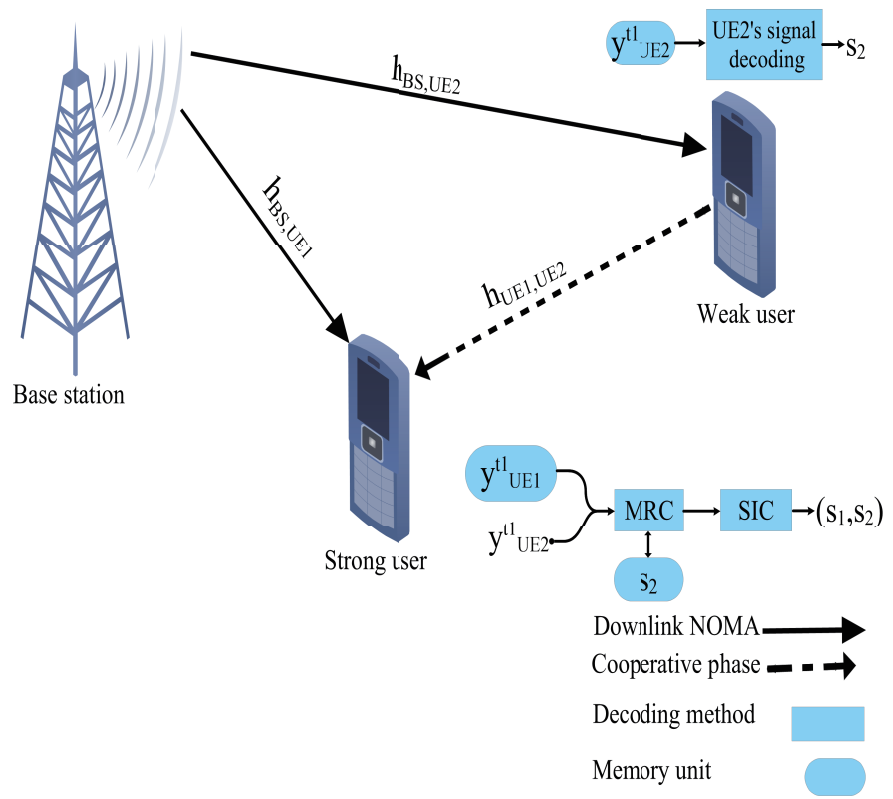

Figure 2: The proposed DC-NOMA scheme with one BS and two users.

As shown in Fig. 2, downlink phase occurs from the base-station to the users and cooperative transmission takes place between the two users. The base station and the two users share a single antenna and work in half-duplex mode [27]. Hereafter, subscripts BS, $\mathrm{UE}_{1}, \mathrm{UE}_{2}$ denote to the base-station, user one, and user two respectively. Also, the time indexes $t_{1}$ and $t_{2}$ denote to the first time slot and second time slot respectively.

$h_{B S, U E 1}$ and $h_{B S, U E 2}$ are the coefficient of channels between the base station and users one and two, respectively, and $h_{U E 1, U E 2}$ is the channel coefficient between both users. For easy signal detection, the channel state information (CSI) is assumed to be available at the receiver while only statistical CSI is available at the transmitter [26]. The block of transmission denotes here to the time slot, where each block represents one time slot [28]. In addition,the channel is supposed to be a rayleigh fading channels $h_{\delta}$ in which the distribution is followed the circularly symmetric complex Gaussian distribution with zero-mean and variance $\beta_{\delta}$ distributions for the channel coefficient power are given by $[26]$

$$
f_{\left|h_{\delta}\right|^{2}}=\frac{1}{\beta_{\delta}} \exp \left(-\frac{x}{\beta_{\delta}}\right), x \geq 0
$$

and

$$
F_{\left|h_{\delta}\right|^{2}}=1-\exp \left(-\frac{x}{\beta_{\delta}}\right)
$$

respectively, where $\left|h_{\delta}\right|^{2}$ is the channel gain of link $\delta$. In addition, the strong user is the user that performs successive interference cancellation (SIC), where the classification of the strong users and weak users depends on the value of $h_{\delta}[29]$. Therefore, the user that has larger $h_{\delta}$ is considered as the strong user and the user that has small $h_{\delta}$ is considered as the weak user. In contrast, the optimal SIC can't be achieved in our scheme because the transmitter has only statistical SCI [30]. Thus, we use the sup-optimal SIC in order to distinguish between the users. Without loss of generality, the strong user is the user that has a larger $\beta_{\delta}$ and the weak user is the one that has the smaller $\beta_{\delta}$. In other words, when the condition $\beta_{B S, U E 1}>\beta_{B S, U E 2}$ is satisfied that mean that the strong user is 
$\mathrm{UE}_{1}$ and the weak user is $\mathrm{UE}_{2}$. The sub-optimal SIC occurs when the condition $\beta_{B S, U E 1}>\beta_{B S, U E 2}$ is satisfied, but that is not guarantee to meet $\left|h_{B S, U E 1}\right|^{2}>\left|h_{B S, U E 2}\right|^{2}$. However, Under statistical CSI, it is a simple and efficient technique [31]. In NOMA scheme, there are two criteria in terms of transmitting power and fairness which are used to formulated two optimization problems, subjected to outage probability and the optimal decoding order [32]. In our scheme, we follow a similar, yet different approach to the scheme in [26] to get improvement in the system performance. In other words, the user with the weak channel conditions $\left(\mathrm{UE}_{2}\right)$ acts as a relay to assist the strong user.

As shown in Fig. 2, our proposed scheme has two phases. The first phase is the downlink phase (i.e., first time slot) and the second phase is the cooperative phase (i.e., second time slot), both phases represent by time slots and have same time duration.

\subsection{The Proposed DC-NOMA Scheme}

\subsubsection{First Phase: Downlink Phase}

During the first time slot (i.e., downlink phase), the BS transmits a superimposed signal to $U E_{1}$ and $U E_{2}$ as follows:

$$
x_{B S}^{t_{1}}=\sqrt{\alpha_{U E 1}^{t_{1}} P_{0}} s_{1}+\sqrt{\alpha_{U E 2}^{t_{1}} P_{0}} s_{2}
$$

where $P_{0}$ is the transmit power from the source $(B S), s_{1}$ and $s_{2}$ are the data symbols for $\mathrm{UE}_{1}$ and $\mathrm{UE}_{2}$ respectively. The parameters $\alpha_{U E 1}^{t_{1}}$ and $\alpha_{U E 2}^{t_{1}}$ denote the power allocation factors for $\mathrm{UE}_{1}$ and $\mathrm{UE}_{2}$ respectively, and $t_{1}$ denote the first time slot. In our proposed scheme, we allocate more power to the weak user in order to achieve some measure of fairness, for that we should achieve the condition $\alpha_{U E 1}^{t_{1}} \leq \alpha_{U E 2}^{t_{1}}$ and $\alpha_{U E 1}^{t_{1}}+\alpha_{U E 2}^{t_{1}}=1$. In the users side, $\mathrm{UE}_{1}$ and $\mathrm{UE}_{2}$ are ready to receive a superimposed signal from the BS. Thus, the received signals at both users are given as follows:

$$
\begin{aligned}
y_{U E_{1}}^{t_{1}} & =h_{B S, U E 1} x_{B S}^{t_{1}}+z_{U E 1}^{t_{1}} \\
& =h_{B S, U E 1}\left(\sqrt{\alpha_{U E 1}^{t_{1}} P_{0}} s_{1}+\sqrt{\alpha_{U E 2}^{t_{1} P_{0}} s_{2}}\right)+z_{U E 1}^{t_{1}}
\end{aligned}
$$

and

$$
\begin{aligned}
y_{U E_{2}}^{t_{1}} & =h_{B S, U E 2} x_{B S}^{t_{1}}+z_{U E 2}^{t_{1}} \\
& =h_{B S, U E 2}\left(\sqrt{\alpha_{U E 1}^{t_{1}} P_{0}} s_{1}+\sqrt{\alpha_{U E 2}^{t_{1} P_{0}} s_{2}}\right)+z_{U E 2}^{t_{1}}
\end{aligned}
$$

where $z_{U E 1}^{t_{1}} \sim C N\left(0, \sigma^{2}\right)$ and $z_{U E 2}^{t_{1}} \sim C N\left(0, \sigma^{2}\right)$ are the complex additive white Gaussian noise (AWGN) at $\mathrm{UE}_{1}$ and $\mathrm{UE}_{2}$ respectively.Note that noise power $\sigma^{2}$ is the same for both users.

In this paper, we follow a similar, yet different approach to the scheme in [26]. In the first phase, when $\mathrm{UE}_{1}$ receives the superimposed signal, it will keep this signal in the memory without decoding and will wait for the next phase operations (i.e. cooperative phase operation). Meanwhile, $\mathrm{UE}_{2}$ decodes its message by considering $\mathrm{UE}_{1}$ message as noise. Thereby, the SINR for $\mathrm{UE}_{2}$ to decode its own message is given by:

$$
S I N R_{U E_{2}}^{t_{1}}=\frac{\rho\left|h_{B S, U E_{2}}\right|^{2} \alpha_{U E_{2}}^{t_{1}}}{\rho\left|h_{B S, U E_{1}}\right|^{2} \alpha_{U E_{1}}^{t_{1}}+1}
$$

where $\rho=\frac{P_{0}}{\sigma^{2}}$ is the transmit signal to noise ratio. Note that $\mathrm{UE}_{1}$ and $\mathrm{UE}_{2}$ transmit their signals at constant downlink rates $\left(R_{U E_{1}}^{D L}\right)$ and $\left(R_{U E_{2}}^{D L}\right)$ respectively. In order to decode $s_{2}$ message of $\mathrm{UE}_{2}$, the achievable data rate must be at least equal the target data rate $\left(R_{U E_{2}}^{D L}\right)$. Therefore, the achievable data rate of $\mathrm{UE}_{2}$ for the first phase is given by:

$$
R_{U E_{2}} \leqslant C_{U E_{2}}=\frac{1}{2} \log _{2}\left(1+S I N R_{U E_{2}}^{t_{1}}\right)
$$

\subsubsection{Second Phase: Cooperative Phase}

As mentioned previously, the decoding operation for $\mathrm{UE}_{2}$ message occurred in the first time slot. Consequently, $\mathrm{UE}_{2}$ will broadcast its decoded signal $s_{2}$ to $\mathrm{UE}_{1}$. Therefore, the transmitted signal from $\mathrm{UE}_{2}$ to $\mathrm{UE}_{1}$ in the second time slot is given by:

$$
x_{U E_{2}}^{t_{2}}=\sqrt{\alpha_{U E 1}^{t_{2} P_{0}}} s_{2}
$$


where $\alpha_{U E 1}^{t_{2}}$ is the power allocation factor for UE1 at $t_{2}$. As a result, the received signal at $\mathrm{UE}_{1}$ is given by:

$$
\begin{aligned}
y_{U E_{1}}^{t_{2}} & =h_{U E 1, U E 2} x_{U E_{2}}^{t_{2}}+z_{U E 1}^{t_{2}} \\
& =h_{U E 1, U E 2} \sqrt{\alpha_{U E 1}^{t_{2}} P_{0}} s_{2}+z_{U E 1}^{t_{2}}
\end{aligned}
$$

We note that the BS usually is un-limited in power, whereas the users are power-limited. Thus, we can increase the power allocation coefficient factors to $\mathrm{UE}_{2}$ to enhance its performance and thus increase the SINR for $\mathrm{UE}_{2}$. Furthermore, we can improve the decoding of $\mathrm{UE}_{2}$ message $\left(s_{2}\right)$ at $\mathrm{UE}_{1}$ due to the cooperation between the two users. Accordingly, we can increase the SINR of the strong user $\left(\mathrm{UE}_{1}\right)$ to achieve higher channel efficiency through cooperation with $\mathrm{UE}_{2}$. Noting that, the power allocation factors can be controlled by either increasing or decreasing its value to improve the spectral efficiency in both users.

In our scheme, we note that $\mathrm{UE}_{1}$ has two independent received signals for $s_{2}$, one signal comes from the BS at the first time slot which is $y_{U E_{1}}^{t_{1}}$ and the other signal comes from $\mathrm{UE}_{2}$ at the cooperative phase which is $y_{U E_{1}}^{t_{2}}$, each signal has different weight. Therefore, to apply the SIC at $\mathrm{UE}_{1}$ with the two signals, $\mathrm{UE}_{1}$ will use maximum ratio combining(MRC) to decode the $s_{2}$ message $[33,34]$. Therefore, the SINR for $\mathrm{UE}_{1}$ to decode $\mathrm{UE}_{2}$ message with MRC is given by:

$$
S I N R_{U E_{1}, U E_{2} \rightarrow M R C}^{t_{1}, t_{2}}=S I N R_{U E 1, U E 2}^{t_{1}}+S I N R_{U E 1, U E 2}^{t_{2}}
$$

where $S I N R_{U E 1, U E 2}^{t_{1}}$ is the SINR for $\mathrm{UE}_{1}$ to decode the message of $\mathrm{UE}_{2}$ at $t_{1}$, which given by:

$$
S I N R_{U E 1, U E 2}^{t_{1}}=\frac{\rho\left|h_{B S, U E_{1}}\right|^{2} \alpha_{U E_{2}}^{t_{1}}}{\rho\left|h_{B S, U E_{1}}\right|^{2} \alpha_{U E_{1}}^{t_{1}}+1}
$$

and $S I N R_{U E 1, U E 2}^{t_{2}}$ is the SINR for $\mathrm{UE}_{1}$ to decode the $\mathrm{UE}_{2}$ message at $t_{2}$, which is given by :

$$
S I N R_{U E 1, U E 2}^{t_{2}}=\rho\left|h_{U E_{1}, U E_{2}}\right|^{2} \alpha_{U E_{1}}^{t_{2}}
$$

The message $s_{2}$ cannot be decoded and cancelled at $U E_{1}$ except if the following condition is satisfied, which is given as:

$$
\frac{1}{2} \log _{2}\left(1+S I N R_{U E_{1}, U E_{2} \rightarrow M R C}^{t_{1}, t_{2}}\right) \geq R_{U E_{2}}^{D L}
$$

where $R_{U E_{2}}^{D L}$ is the target data rate of downlink transmission of $\mathrm{UE}_{2}$. In contrast, if the above condition in Equation (13) is not satisfied, then the SIC process will be failed. Meanwhile, if the SIC process fails, $\mathrm{UE}_{1}$ cannot decode its message $s_{1}$. Furthermore, with a successful SIC process, $U E_{1}$ can decode its message by subtract the message of $\mathrm{UE}_{2}$ from its observation $y_{U E_{1}}^{t_{1}}$, therefore, the SINR for $\mathrm{UE}_{1}$ to decode its message $s_{1}$ is given by

$$
S I N R_{U E 1}^{t_{2}}=\rho\left|h_{B S, U E 1}\right|^{2} \alpha_{U E_{1}}^{t_{1}}
$$

Then, the achievable rate at $\mathrm{UE}_{1}$ is given by

$$
C_{U E_{1}}=\frac{1}{2} \log _{2}\left(1+S I N R_{U E_{1}}^{t_{2}}\right)
$$

\subsection{Performance Analysis}

In this section, the outage performance, throughput, and diversity order of the proposed DC-NOMA scheme are investigated. These three metrics are used to characterize the spectral efficiency of our proposed scheme.

The outage probability $\left(P_{\text {out }}\right)$ is the probability that the capacity of the channel $(\mathrm{C})$ fails to satisfy its pre-defined threshold (R). Thus, the outage probability can be expressed mathematically as follows:

$$
P_{\text {out }}=\operatorname{Pr}(C<R)=\operatorname{Pr}\left(\log _{2}(1+S I N R)<R\right)
$$

where $C$ is the channel capacity and $R$ is the target data rate. For a given data rate, the outage probability becomes a crucial metric in the analysis process. For that, we analyze this outage for 
each link in accordance with (QoS) requirements of each user. Furthermore, We can obtain the diversity order for each path to characterize the spectral efficiency in the proposed scheme. In addition, the system outage throughput is calculated to show the increase in spectral efficiency.

In the given scheme, the target data rates for the downlink transmission of $\mathrm{UE}_{1}$ and $\mathrm{UE}_{2}$ are adjusted according to the channels conditions of these users. For example, if the achievable data rates for $\mathrm{UE}_{1}$ and $\mathrm{UE}_{2}$ in our scheme are greater or equal the target data rates $R_{U E_{1}}^{D L}$ and $R_{U E_{2}}^{D L}$ respectively, then we successfully decode the signals for both users. Otherwise, the proposed scheme will fail to decode the signals of both users, and thus the outage of the system completely occurs.

\subsubsection{Outage Probability}

To measure the performance of our proposed scheme in the wireless communication system, we will derive the outage probability of downlink transmission for $\mathrm{UE}_{1}$ and $\mathrm{UE}_{2}$ in this sub-section. For notational convenience, we define $\gamma_{U E_{1}}^{D L}=2 R_{U E_{1}}^{D L}-1$, and $\gamma_{U E_{2}}^{D L}=2 R_{U E_{2}}^{D L}-1$ to be the corresponding SNR for each given data rate.

At the beginning of our analysis, We will derive the outage probability of $\mathrm{UE}_{2}$. Outage occurs when $\mathrm{UE}_{2}$ fails to decode its message $\left(s_{2}\right)$. For that, the outage probability of downlink transmission for $\mathrm{UE}_{2}$ is derived as follows:

$$
P_{\text {out }}^{U E_{2} D L}=\operatorname{Pr}\left\{\frac{1}{2} \log _{2}\left(1+S I N R_{U E_{2}}^{t_{1}}\right)<R_{U E_{2}}^{D L}\right\}
$$

After some manipulations, we substitute $R_{U E_{2}}^{D L}=\frac{1}{2} \log _{2}\left(1+\gamma_{U E_{2}}^{D L}\right)$ into Equation (17) to obtain the following result:

$$
P_{\text {out }}^{U E_{2} D L}=\left\{S I N R_{U E_{2}}^{t_{1}}<\gamma_{U E_{2}}^{D L}\right\}
$$

Substituting Equation (6) in Equation (18) as follows:

$$
\begin{aligned}
P_{\text {out }}^{U E_{2}, D L} & =\operatorname{Pr}\left\{\frac{\rho\left|h_{B S, U E_{2}}\right|^{2} \alpha_{U E_{2}}^{t_{1}}}{\rho\left|h_{B S, U E_{1}}\right|^{2} \alpha_{U E_{1}}^{t_{1}}+1}<\gamma_{U E_{2}}^{D L}\right\} \\
& =\operatorname{Pr}\left\{\left|h_{B S, U E_{2}}\right|^{2}<\frac{\gamma_{U E_{2}}^{D L}}{\left(\alpha_{U E_{2}}^{t_{1}}-\gamma_{U E_{2}}^{D L} \alpha_{U E_{1}}^{t_{1}}\right) \rho}\right\}
\end{aligned}
$$

Then, we use the CDF of channel coefficient $h_{B S, U E_{2}}$ to express the outage probability for $\mathrm{UE}_{2}$ in the following form:

$$
P_{o u t}^{U E_{2} D L}=1-\exp \left(\frac{-\gamma_{U E_{2}}^{D L}}{\left(\alpha_{U E_{2}}^{t_{1}}-\gamma_{U E_{2}}^{D L} \alpha_{U E_{1}}^{t_{1}}\right)} \frac{1}{\rho \beta_{B S, U E_{2}}}\right)
$$

The result in Equation (20) is only satisfied when when $\gamma_{U E_{2}}^{D L}<\frac{\alpha_{U E 2}^{t_{1}}}{\alpha_{U E 1}^{t_{1}}}$, otherwise, the outage probability is always one.

As for $\mathrm{UE}_{1}$, the outage probability is given as:

$$
\begin{aligned}
P_{\text {out }}^{U E_{1} D L}= & \operatorname{Pr}\left\{\frac{1}{2} \log _{2}\left(1+S I N R_{U E_{1}, U E_{2} \rightarrow M R C}^{t_{1}, t_{2}}\right)<R_{U E_{2}}^{D L}\right\} \\
& +\operatorname{Pr}\left\{\frac{1}{2} \log _{2}\left(1+S I N R_{U E_{1}, U E_{2} \rightarrow M R C}^{t_{1}, t_{2}}\right) \geq R_{U E_{2}}^{D L},\right. \\
& \frac{1}{2} \log _{2}\left(1+S I N R_{U E 1}^{t_{1}}\right)<R_{U E_{1}}^{D L}
\end{aligned}
$$

We note that the outage for $\mathrm{UE}_{1}$ occurs at two cases,(1) when $\mathrm{UE}_{2}$ fails to decode MRC signal $s_{2}^{\prime}$, (2) when $\mathrm{UE}_{1}$ fails to decode its message $\left(s_{1}\right)$ even if $\mathrm{UE}_{2}$ successes to decode its message. In addition, each event in the outage probability is independent of other events, so we can rewrite the 
above equation in the following form:

$$
\begin{aligned}
P_{\text {out }}^{U E_{1} D L}= & \operatorname{Pr}\left\{\frac{1}{2} \log _{2}\left(1+S I N R_{U E_{1}, U E_{2} \rightarrow M R C}^{t_{1}, t_{2}}\right)<R_{U E_{2}}^{D L}\right\} \\
& +\operatorname{Pr}\left\{\frac{1}{2} \log _{2}\left(1+S I N R_{U E_{1}, U E_{2} \rightarrow M R C}^{t_{1}, t_{2}}\right) \geq R_{U E_{2}}^{D L}\right\} \\
& \operatorname{Pr}\left\{\frac{1}{2} \log _{2}\left(1+S I N R_{U E 1}^{t_{1}}\right)<R_{U E_{1}}^{D L}\right\}
\end{aligned}
$$

based on Equation (22), we can define $Q_{2}$ as follows:

$$
Q_{2}=\operatorname{Pr}\left\{\frac{1}{2} \log _{2}\left(1+S I N R_{U E 1}^{t_{1}}\right)<R_{U E_{1}}^{D L}\right\}
$$

To deriving $Q_{2}$, we substitute $R_{U E_{1}}^{D L}=\frac{1}{2} \log _{2}\left(1+\gamma_{U E_{1}}^{D L}\right)$, and Equation (14) in Equation (23) to obtain the following:

$$
\begin{aligned}
Q_{2} & =\operatorname{Pr}\left\{\rho\left|h_{B S, U E 1}\right|^{2} \alpha_{U E_{1}}^{t_{1}}<\gamma_{U E_{1}}^{D L}\right\} \\
& =\operatorname{Pr}\left\{\left|h_{B S, U E 1}\right|^{2}<\frac{\gamma_{U E_{1}}^{D L}}{\rho \alpha_{U E_{1}}^{t_{1}}}\right\}
\end{aligned}
$$

Again, by applying the CDF of $\left|h_{B S, U E 1}\right|^{2}$ in the above equation, we can simplify $Q_{2}$ as:

$$
Q_{2}=1-\exp \left\{\frac{-\gamma_{U E_{1}}^{D L}}{\rho \alpha_{U E_{1}}^{t_{1}} \beta_{B S, U E_{1}}}\right\}
$$

According to Equation (22), we can also express $Q_{1}$ as follows:

$$
Q_{1}=\operatorname{Pr}\left\{\frac{1}{2}\left(1+S I N R_{U E_{1}, U E_{2} \rightarrow M R C}^{t_{1}, t_{2}}\right)<R_{U E_{2}}^{D L}\right\}
$$

To evaluate $Q_{1}$, we first derive the distributions of $S I N R_{U E 1, U E 2}^{t_{1}}$ and $S I N R_{U E 1, U E 2}^{t_{2}}$ respectively. Again, we use a similar procedure that used in $Q_{2}$ to applying it in $Q_{1}$. Thus, we can obtain the following result:

$$
Q_{1}=\operatorname{Pr}\left\{\left(\frac{\rho\left|h_{B S, U E_{1}}\right|^{2} \alpha_{U E_{2}}^{t_{1}}}{\rho\left|h_{B S, U E_{1}}\right|^{2} \alpha_{U E_{1}}^{t_{1}}+1}+\rho\left|h_{U E_{1}, U E_{2}}\right|^{2} \alpha_{U E_{1}}^{t_{2}}\right)<\gamma_{2}^{D L}\right\}
$$

Now, we apply the double integration for $Q_{1}$ as follows:

$$
Q_{1}=\iint_{A} f_{S I N R_{U E 1, U E 2}^{t_{1}}}(x) f_{S I N R_{U E 1, U E 2}^{t_{2}}}(y) d y d x
$$

where A correspond to the event $S I N R_{U E 1, U E 2}^{t_{1}}+S I N R_{U E 1, U E 2}^{t_{2}}<\gamma_{U E_{2}}^{D L}$. To find the PDF for $S I N R_{U E 1, U E 2}^{t_{1}}$, we first give the CDF expression of $S I N R_{U E 1, U E 2}^{t_{1}}$ as follows:

$$
F_{S I N R_{U E 1, U E 2}^{t_{1}}}(x)=\operatorname{Pr}\left\{S I N R_{U E 1, U E 2}^{t_{1}}<x\right\}
$$

where $0<x<\frac{\alpha_{U E_{2}}^{t 1}}{\alpha_{U E_{1}}^{t 1}}$. Thus, the CDF and PDF of SINR for $\mathrm{UE}_{1}$ to decode the messsage of $\mathrm{UE}_{2}$ at the first phase $\left(t_{1}\right)$ are given by:

$$
\begin{aligned}
& F_{S I N R_{U E 1, U E 2}^{t_{1}}(x)}=F_{\left|h_{B S, U E_{1}}\right|^{2}}\left(\frac{x}{\rho\left(\alpha_{U E_{2}}^{t_{1}}-x \alpha_{U E_{1}}^{t_{1}}\right)}\right) \\
& f_{S I N R_{U E 1, U E 2}^{t_{1}}(x)}=\frac{\alpha_{U E_{2}}^{t_{1}}}{\rho\left(\alpha_{U E_{2}}^{t_{1}}-x \alpha_{U E_{1}}^{t_{1}}\right)^{2}} f_{\left|h_{B S, U E_{1}}\right|^{2}}\left(\frac{x}{\rho\left(\alpha_{U E_{2}}^{t_{1}}-x \alpha_{U E_{1}}^{t_{1}}\right)}\right)
\end{aligned}
$$


respectively. By following the same procedure, we can obtain the $\mathrm{CDF}$ and $\mathrm{PDF}$ of $S I N R$ for $\mathrm{UE}_{1}$ to decode the messsage of $\mathrm{UE}_{2}$ at the second phase $\left(t_{2}\right)$ for $0<y<\gamma_{U E_{2}}^{D L}$ as follows:

$$
\begin{aligned}
& F_{S I N R_{U E 1, U E 2}^{t_{2}}(y)}=F_{\left|h_{U E_{1}, U E_{2}}\right|^{2}}\left(\frac{y}{\rho \alpha_{U E_{1}}^{t_{2}}}\right) \\
& f_{S I N R_{U E 1, U E 2}^{t_{2}}(y)}=\frac{1}{\rho \alpha_{U E_{1}}^{t_{2}}} f_{S I N R_{U E 1, U E 2}^{t_{2}}}\left(\frac{y}{\rho \alpha_{U E_{1}}^{t_{2}}}\right)
\end{aligned}
$$

respectively. Then, substituting equations(31), and (33) into Equation (28) to obtain the following:

$$
\begin{aligned}
Q_{1}= & \int_{0}^{\phi_{1}} \int_{0}^{\gamma_{U E_{2}}^{D L}-x} \frac{1}{\rho \alpha_{U E_{1}}^{t_{2}}} \\
& f_{\left|h_{U E_{1}, U E_{2}}\right|^{2}}\left(\frac{y}{\rho \alpha_{U E_{1}}^{t_{2}}}\right) \frac{\alpha_{U E_{2}}^{t_{1}}}{\rho\left(\alpha_{U E_{2}}^{t_{1}}-x \alpha_{U E_{1}}^{t_{1}}\right)^{2}} \\
& f_{\left|h_{B S, U E_{1}}\right|^{2}}\left(\frac{x}{\rho\left(\alpha_{U E_{2}}^{t_{1}}-x \alpha_{U E_{1}}^{t_{1}}\right)}\right) d y d x
\end{aligned}
$$

where $\phi_{1}=\min \left(\gamma_{U E_{2}}^{D L}, \frac{\alpha_{U E 2}^{t_{1}}}{\alpha_{U E 1}^{t_{1}}}\right)$. Since $\left|h_{B S, U E_{1}}\right|^{2}$ and $\left|h_{U E_{1}, U E_{2}}\right|^{2}$ are independent, we can further integrate each part separately. Lets define B as follows:

$$
B=\int_{0}^{\gamma_{U E_{2}}^{D L_{2}}-x} \frac{1}{\rho \alpha_{U E_{1}}^{t_{2}}} f_{\left|h_{U E_{1}, U E_{2}}\right|^{2}}\left(\frac{y}{\rho \alpha_{U E_{1}}^{t_{2}}}\right) d y
$$

Thus, we can integrate $\mathrm{B}$ to obtain the following result:

$$
B=1-\exp \left(-\frac{\gamma_{U E_{2}}^{D L}-x}{\rho \alpha_{U E_{1}}^{t_{2}} \beta_{U E_{1}, U E_{2}}}\right)
$$

So, by substituting Equation (36) into Equation (34), we can complete the integration of $Q_{1}$ as follows:

$$
\begin{aligned}
Q_{1}= & \left.\int_{0}^{\phi_{1}}\left(1-\exp \left(\frac{x-\gamma_{U E_{2}}^{D L}}{\rho \beta_{U E_{1}, U E 2} \alpha_{U E_{1}}^{t_{2}}}\right)\right) \frac{\alpha_{U E_{2}}^{t_{1}}}{\rho \beta_{B S, U E_{1}}\left(\alpha_{U E_{2}}^{t_{1}}-x \alpha_{U E_{1}}^{t_{1}}\right)^{2}}\right) d x \\
& \cdot \exp \left(\frac{-x}{\rho \beta_{B S, U E_{1}}\left(\alpha_{U E_{2}}^{t_{1}}-x \alpha_{U E_{1}}^{t_{1}}\right)}\right) d x-\frac{\alpha_{U E 2}^{t_{1}}}{\rho \beta_{B S, U E_{1}}} \\
= & \int_{0}^{\phi_{1}} \frac{\alpha_{U E_{2}}^{t_{1}} \frac{-x}{\rho \beta_{B S, U E_{1}}\left(\alpha_{U E_{2}}^{t_{1}}-x \alpha_{U E_{1}}^{t_{1}}\right)^{2}}}{} \\
& \exp \left(\frac{1}{\rho \beta_{B S, U E_{1}}\left(\alpha_{U E_{2}}^{t_{1}}-x \alpha_{U E_{1}}^{t_{1}}\right)}\right) d x \\
& \int_{0}^{\phi_{1}} \frac{1}{\left(\alpha_{U E_{2}}^{t_{1}}-x \alpha_{U E_{1}}^{t_{1}}\right)^{2}}{ }^{2} \\
& \exp \left(-\frac{\gamma_{U E_{2}}^{D L}-x}{\rho \beta_{U E_{1}, U E 2} \alpha_{U E_{1}}^{t_{2}}}-\frac{x}{\rho \beta_{B S, U E_{1}}\left(\alpha_{U E_{2}}^{t_{1}}-x \alpha_{U E_{1}}^{t_{1}}\right)}\right) d x
\end{aligned}
$$

The above equation can be simplified as

$$
Q_{1}=F_{S I N R_{U E 1, U E 2}^{t_{1}}}\left(\phi_{1}\right)-\frac{\alpha_{U E 2}^{t_{1}}}{\rho \beta_{B S, U E_{1}}} C
$$


Where the first term in Equation (37) is the CDF of $S I N R_{U E 1, U E 2}^{t_{1}}$, which given as :

$$
F_{S I N R_{U E 1, U E 2}^{t_{1}}}\left(\phi_{1}\right)=1-\exp \left(\frac{-\phi_{1}}{\rho \beta_{B S, U E 1}\left(\alpha_{U E_{2}}^{t_{1}}-\phi_{1} \alpha_{U E_{1}}^{t_{1}}\right)}\right)
$$

Defining $C$ in the second term of Equation (37)as follows:

$$
\begin{aligned}
C= & \int_{0}^{\phi_{1}} \frac{1}{\left(\alpha_{U E_{2}}^{t_{1}}-x \alpha_{U E_{1}}^{t_{1}}\right)^{2}} \\
& \exp \left(-\frac{\gamma_{U E_{2}}^{D L}-x}{\rho \beta_{U E_{1}, U E 2} \alpha_{U E_{1}}^{t_{2}}}-\frac{x}{\rho \beta_{B S, U E_{1}}\left(\alpha_{U E_{2}}^{t_{1}}-x \alpha_{U E_{1}}^{t_{1}}\right)}\right) d x
\end{aligned}
$$

This integration is more complicated to derive it directly. So, to achieve the final result of $P_{\text {out }}^{U E_{1}, D L}$, we apply the Gauss-Chebyshev integration [35] to obtain $C$ via a closed-form approximation as follows:

$$
C=\frac{\phi_{1}}{m} \sum_{i=1}^{m} \frac{\pi}{m}|\sin (w)| f\left(l_{i}\right)
$$

where $m$ is the number of Gauss-Chebyshev integral approximation terms [35], $w=\frac{2 i-1}{2 m} \pi$, $l_{i}=\frac{\phi_{1}}{2}+\frac{\phi_{1}}{2} \cos (w)$ and $f(x)$ is a function which is given by

$$
\begin{aligned}
f(x)= & \frac{1}{\left(\alpha_{U E_{2}}^{t_{1}}-x \alpha_{U E_{1}}^{t_{1}}\right)^{2}} \\
& \exp \left(-\frac{\gamma_{U E_{2}}^{D L}-x}{\rho \beta_{U E_{1}, U E 2} \alpha_{U E_{1}}^{t_{2}}}-\frac{x}{\rho \beta_{B S, U E_{1}}\left(\alpha_{U E_{2}}^{t_{1}}-x \alpha_{U E_{1}}^{t_{1}}\right)}\right)
\end{aligned}
$$

Now, substituting Equations (39) and (41) into Equation (38) to obtain $Q_{1}$ as follows:

$$
Q_{1}=\left(1-\exp \left(\frac{-\phi_{1}}{\rho \beta_{B S, U E 1}\left(\alpha_{U E_{2}}^{t_{1}}-\phi_{1} \alpha_{U E_{1}}^{t_{1}}\right)}\right)\right)-\frac{\alpha_{U E 2}^{t_{1}} \phi_{1}}{2 \rho \beta_{B S, U E_{1}}} \sum_{i=1}^{m} \frac{\pi}{m}|\sin (w)| f\left(l_{i}\right)
$$

Substituting $Q_{1}$ and $Q_{2}$ into Equation (22) to obtain $P_{\text {out }}^{U E_{1}, D L}$ as follows:

$$
\begin{aligned}
P_{\text {out }}^{U E_{1} D L}= & 1-\exp \left(\frac{-\phi_{1}}{\rho \beta_{B S, U E 1}\left(\alpha_{U E_{2}}^{t_{1}}-\phi_{1} \alpha_{U E_{1}}^{t_{1}}\right)}\right)-\frac{\alpha_{U E 2}^{t_{1}} \phi_{1}}{2 \rho \beta_{B S, U E_{1}}} \sum_{i=1}^{m} \frac{\pi}{m}|\sin (w)| f\left(l_{i}\right) \\
& +\left(\exp \left(\frac{-\phi_{1}}{\rho \beta_{B S, U E 1}\left(\alpha_{U E_{2}}^{t_{1}}-\phi_{1} \alpha_{U E_{1}}^{t_{1}}\right)}\right)+\frac{\alpha_{U E 2}^{t_{1}} \phi_{1}}{2 \rho \beta_{B S, U E_{1}}} \sum_{i=1}^{m} \frac{\pi}{m}|\sin (w)| f\left(l_{i}\right)\right) \\
& \left(1-\exp \left(\frac{-\gamma_{U E_{1}}^{D L}}{\rho \alpha_{U E_{1}}^{t_{1}} \beta_{B S, U E_{1}}}\right)\right)
\end{aligned}
$$

which can be simplified as:

$$
\begin{aligned}
P_{\text {out }}^{U E_{1} D L}= & 1-\exp \left(\frac{-\gamma_{U E_{1}}^{D L}}{\rho \alpha_{U E_{1}}^{t_{1}} \beta_{B S, U E_{1}}}\right) \exp \left(\frac{-\phi_{1}}{\rho \beta_{B S, U E 1}\left(\alpha_{U E_{2}}^{t_{1}}-\phi_{1} \alpha_{U E_{1}}^{t_{1}}\right)}\right) \\
& -\exp \left(\frac{-\gamma_{U E_{1}}^{D L}}{\rho \alpha_{U E_{1}}^{t_{1}} \beta_{B S, U E_{1}}}\right) \frac{\alpha_{U E 2}^{t_{1}} \phi_{1}}{2 \rho \beta_{B S, U E_{1}}} \sum_{i=1}^{m} \frac{\pi}{m}|\sin (w)| f\left(l_{i}\right)
\end{aligned}
$$

The above result for $P_{o u t}^{U E_{1}, D L}$ is only non-trivial if $\left(\alpha_{U E_{2}}^{t_{1}}-\gamma_{U E_{1}^{D L}} \alpha_{U E_{1}}^{t_{1}}\right)>0$, otherwise, $P_{\text {out }}^{U E_{1}, D L}$ is always one. 


\subsubsection{Diversity Order}

in this subsection, to gain a better understanding of the system outage efficiency, we examine the diversity order for each link. The diversity order can be defined as the slope of the bit error rate (BER), the capacity, or the outage probability as SNR gets higher. So that, we can measure the diversity order when the SNR is very large [36]. Therefore, the mathematical definition of diversity order is given by:

$$
D=\lim _{\rho \rightarrow \infty}-\frac{\log P_{\text {out }}}{\log \rho}
$$

The achievable diversity order in the DC-NOMA scheme can be obtained by both users. Although $\mathrm{UE}_{1}$ gets signal from $\mathrm{UE}_{2}$ (i.e., $s_{2}^{\prime}$ message), and gets another signal from BS, we can't decide the diversity order value for $\mathrm{UE}_{1}$ directly. At the high $\mathrm{SNR}$ regime, we can use this approximation (i.e., $e^{-x} \approx 1-x$ where $x \rightarrow 0$ ) to obtain the outage probability and the diversity order [21].

To measure the diversity order for $\mathrm{UE}_{2}$, we apply the above approximation to obtain the outage probability of $\mathrm{UE}_{2}$ as follows:

$$
P_{\text {out }}^{U E_{2}, D L}=\frac{\gamma_{U E_{2}}^{D L}}{\left(\alpha_{U E_{2}}^{t_{1}}-\gamma_{U E_{2}}^{D L} \alpha_{U E_{1}}^{t_{1}}\right)} \frac{1}{\rho \beta_{B S, U E_{2}}}
$$

Now, substituting this result in Equation (46) to obtain the diversity order for $\mathrm{UE}_{2}$ as follows:

$$
\begin{aligned}
D_{\text {out }}^{U E_{2}, D L} & =\lim _{\rho \rightarrow \infty}-\frac{\log P_{\text {out }}^{U E_{2}, D L}}{\log \rho} \\
& =\lim _{\rho \rightarrow \infty} \frac{-\log \left(\frac{\gamma_{U E_{2}}^{D L}}{\rho \beta_{B S, U E_{2}}\left(\alpha_{U E_{2}}^{t_{1}}-\gamma_{U E_{2}}^{D L} \alpha_{U E_{1}}^{t_{1}}\right)}\right)}{\log \rho}
\end{aligned}
$$

To simplify this equation, We can use L'Hospital's Rule (i.e., $\left.\lim _{\rho \rightarrow \infty} \frac{f(x)}{g(x)}=\lim _{\rho \rightarrow \infty} \frac{f^{\prime}(x)}{g^{\prime}(x)}\right)$. After some manipulation in this equation, the diversity order can be given as:

$$
D_{\text {out }}^{U E_{2}, D L}=\lim _{\rho \rightarrow \infty} \frac{1 / \rho}{1 / \rho}=1
$$

Therefore, the diversity order of $\mathrm{UE}_{2}$ for the downlink transmission is one. This value is reasonable because we only have one path to transmit $s_{2}$ message from $\mathrm{BS}$ to $\mathrm{UE}_{2}$.

Now, we find the diversity order of $\mathrm{UE}_{1}$ for the downlink transmission by following a similar procedures of $D_{\text {out }}^{U E_{2}, D L}$. First, the outage probability for $\mathrm{UE}_{1}$ at high SNR is given as:

$$
\begin{aligned}
P_{o u t}^{U E_{1}, D L}= & \frac{\phi_{1}}{\rho \beta_{B S, U E 1}\left(\alpha_{U E_{2}}^{t_{1}}-\phi_{1} \alpha_{U E_{1}}^{t_{1}}\right)}+\frac{\gamma_{U E_{1}}^{D L}}{\rho \alpha_{U E_{1}}^{t_{1}} \beta_{B S, U E_{1}}} \\
& -\frac{\gamma_{U E_{1}}^{D L} \phi_{1}}{\rho^{2} \alpha_{U E_{1}}^{t_{1}} \beta_{B S, U E 1}^{2}\left(\alpha_{U E_{2}}^{t_{1}}-\phi_{1} \alpha_{U E_{1}}^{t_{1}}\right)}
\end{aligned}
$$

Substituting Equation (50) into Equation (46) to obtain the diversity order of $\mathrm{UE}_{1}$ as follows:

$$
D_{\text {out }}^{U E_{1}, D L}=\lim _{\rho \rightarrow \infty}-\frac{\log P_{\text {out }}^{U E_{1}, D L}}{\log \rho}
$$

where $P_{o u t}^{U E_{1}, D L}$ is given in Equation (50). Again, by applying L'Hospital's Rule, and after some manipulations in Equation (51), we can obtain the final result of diversity order for $\mathrm{UE}_{1}$ as follows:

$$
D_{\text {out }}^{U E_{1}, D L}=\frac{\frac{\phi_{1}}{\beta_{B S, U E 1}\left(\alpha_{U E_{2}}^{t_{1}}-\phi_{1} \alpha_{U E_{1}}^{t_{1}}\right)}+\frac{\gamma_{U E_{1}}^{D L}}{\alpha_{U E_{1}}^{t_{1}} \beta_{B S, U E_{1}}}}{\frac{\phi_{1}}{\beta_{B S, U E 1}\left(\alpha_{U E_{2}}^{t_{1}}-\phi_{1} \alpha_{U E_{1}}^{t_{1}}\right)}+\frac{\gamma_{U E_{1}}^{D L}}{\alpha_{U E_{1}}^{t_{1}} \beta_{B S, U E_{1}}}}=1
$$


We can note that the diversity order of $\mathrm{UE}_{1}$ is also one because the transmission in cooperative phase occurs for $s_{2}$ only. In addition, the transmission for $\mathrm{UE}_{1}$ message occurs only in a single path which is between BS and this user.

\subsubsection{System Throughput}

The rate of successful information delivery over a wireless communication channel is known as throughput $[37,38]$. Thus, the throughput considers as an important measurement to evaluate the quality of data links. In general, to achieve a maximum rate of throughput, the SINR must be in the optimum level. Therefore, To study the throughput, there are several variables taking into account when we examine it, such as size of packet, transmission rate, received noise power spectral density, received signal power, and channel conditions [39].

In the proposed NOMA scheme, we note that both users transmit their signals at their corresponding data rate. In addition, $\mathrm{UE}_{1}$ can achieve the throughput gain by decoding and cancelling $\mathrm{UE}_{2}$ message before decoding its desired signal [40].

In our scheme, we analyze the throughput according to the outage probabilities and data rates for $\mathrm{UE}_{1}$ and $\mathrm{UE}_{2}$. For that, the mathematical expression of system outage throughput of downlink transmission can be given as:

$$
R=\left(1-P_{\text {out }}^{U E_{1}, D L}\right) R_{U E_{1}}^{D L}+\left(1-P_{\text {out }}^{U E_{2}, D L}\right) R_{U E_{2}}^{D L}
$$

\section{NUMERICAL RESULTS}

Here, the performance of the proposed scheme is discussed by numerical results. The power allocation factors are used to give us flexible control in the amount of power for each user. In downlink NOMA, we compare between our proposed scheme and scheme in [26]. Without loss of generality, The major numerical parameters are listed in Table 1.

Table 1: Numerical parameters for DC-NOMA scheme.

\begin{tabular}{cc}
\hline Parameter & Value \\
\hline \hline channel coefficient variances $\beta_{B S, U E_{1}}$ & 1 \\
\hline channel coefficient variances $\beta_{B S, U E_{2}}$ & 0.05 \\
\hline channel coefficient variances $\beta_{U E_{1}, U E_{2}}$ & 0.8 \\
\hline Power allocation factor $\alpha_{U E_{1}}^{t_{1}}$ & 0.05 \\
\hline Power allocation factor $\alpha_{U E_{2}}^{t_{1}}$ & 0.95 \\
\hline Power allocation factor $\alpha_{U E_{1}}^{t_{2}}$ & 0.01 \\
\hline Target data rate $R_{U E_{1}}^{D L}$ & $0.5,1,1.5 \mathrm{bit} / \mathrm{s} / \mathrm{Hz}$ \\
\hline Target data rate $R_{U E_{2}}^{D L}$ & $0.5,1,1.5 \mathrm{bit} / \mathrm{s} / \mathrm{Hz}$ \\
\hline approximation parameter for Gauss-Chebyshev integration $(m)$ & 100 \\
\hline
\end{tabular}

In our proposed scheme, the operating condition for the DC-NOMA system to determine the outage probabilities is $\alpha_{U E_{2}}^{t_{1}}-\alpha_{U E_{1}}^{t_{1}} \gamma_{U E_{2}}^{D L}>0$. Otherwise, the outage probabilities are always one.

The outage probability versus the normalized average SNR of DC-NOMA system and HDUCNOMA system is presented in Figs. 3 and 4 respectively. Noting that, for a fair comparison, we assume the same duration of time for the frame in the proposed scheme and HDU-CNOMA scheme. Therefore, When the target data rates $R_{1}=R_{2}=1 \mathrm{bit} / \mathrm{s} / \mathrm{Hz}$, we demonstrate the numerical results for our proposed scheme and HDU-CNOMA scheme in this figures. We can observe that the outage probability $\left(P_{\text {out }}^{U E_{1}, D L}\right)$ in Fig. 3 for the proposed scheme outperforms the HDU-CNOMA scheme. Noting that, this improvement in the performance occurs due to not only the transmission of $s_{1}$ from $\mathrm{Bs}$ to $\mathrm{UE}_{1}$, but also $\mathrm{UE}_{1}$ get helps from $\mathrm{UE}_{2}$ in the cooperative phase. For that, we confirm the importance of the cooperation between the two users to increase their SINR's and their spectral efficiency.

Figure 4 illustrates the outage probability for $P_{\text {out }}^{U E_{2}, D L}$ of our proposed scheme compared to that in the HDU-CNOMA scheme at $R_{1}=R_{2}=1 \mathrm{bit} / \mathrm{s} / \mathrm{Hz}$. Note that the improvement in the outage probability for our scheme over the HDU-CNOMA scheme results from the great power that allocated to $\mathrm{UE}_{2}$ from $\mathrm{BS}$ in the downlink phase. In general, the performance of our proposed 


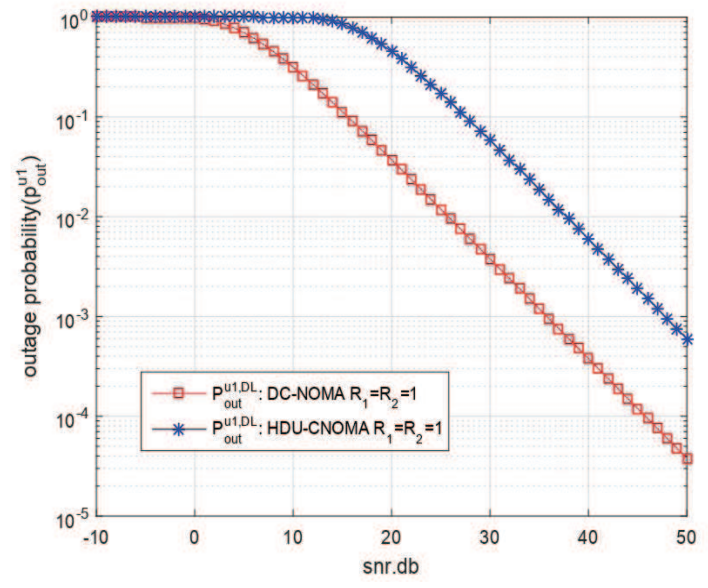

Figure 3: Outage probability of $P_{\text {out }}^{U E_{1}, D L}$ for the proposed DC-NOMA scheme and a conventional CNOMA scheme when $R_{1}=R_{2}=1 \mathrm{bit} / \mathrm{s} / \mathrm{Hz}$.

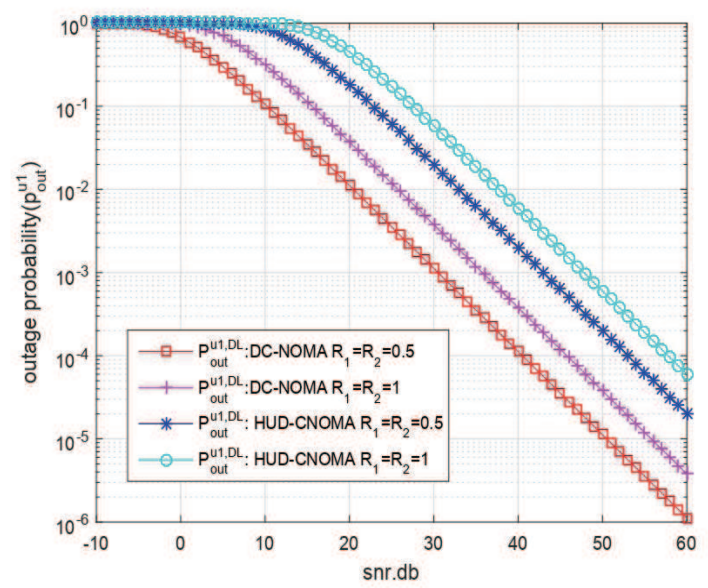

Figure 5: Outage probability of $P_{o u t}^{U E_{1}, D L}$ for the proposed DC-NOMA scheme and a conventional CNOMA scheme when $R_{1}=R_{2}=0.5,1 \mathrm{bit} / \mathrm{s} / \mathrm{Hz}$.

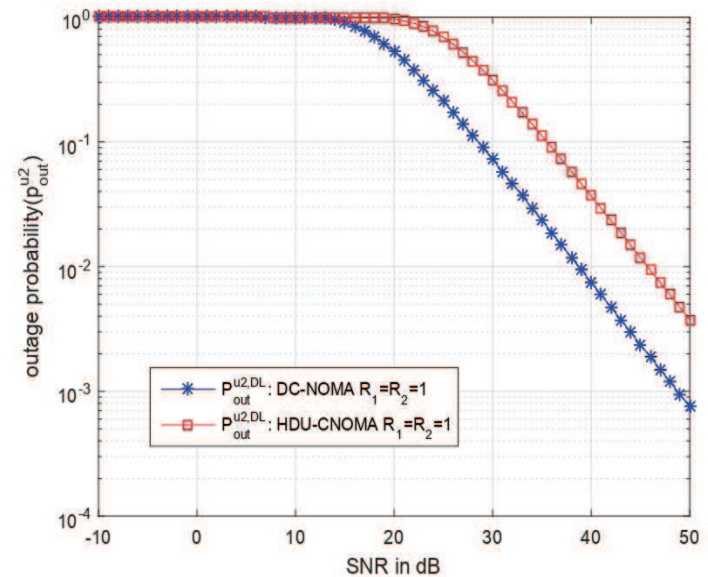

Figure 4: Outage probability of $P_{\text {out }}^{U E_{2}, D L}$ for the proposed DC-NOMA scheme and a conventional CNOMA scheme when $R_{1}=R_{2}=1 \mathrm{bit} / \mathrm{s} / \mathrm{Hz}$.

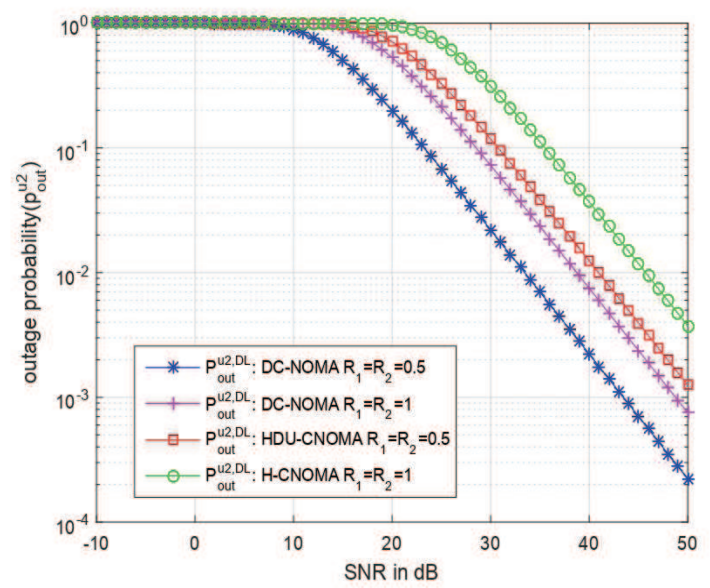

Figure 6: Outage probability of $P_{\text {out }}^{U E_{2}, D L}$ for the proposed DC-NOMA scheme and a conventional CNOMA scheme when $R_{1}=R_{2}=0.5,1 \mathrm{bit} / \mathrm{s} / \mathrm{Hz}$.

scheme in-term of outage probability for both users is better than the that in HDU-CNOMA scheme.

To demonstrate the comparison between the outage probabilities at different data rates, Fig. 5 illustrates that for the outage probability of $\mathrm{UE}_{1}$ when $R_{1}=R_{2}=0.5 \mathrm{bit} / \mathrm{s} / \mathrm{Hz}$ and $R_{1}=R_{2}=$ $1 \mathrm{bit} / \mathrm{s} / \mathrm{Hz}$. We can observe that the outage probability for our proposed scheme at $R_{1}=R_{2}=$ $0.5 \mathrm{bit} / \mathrm{s} / \mathrm{Hz}$ is better than the same outage probability at $R_{1}=R_{2}=1 \mathrm{bit} / \mathrm{s} / \mathrm{Hz}$. In other words, as the target data rate is low as the outage probability is better due to that the capacity of the channel for a user will increase as the target data rate is decrease. On the other hand, the outage probability for HDU-CNOMA scheme will be affected by changing the target data rate values, but the performance of our scheme is still better than the HDU-CNOMA scheme.

Figure 6 illustrates the comparison between the outage probabilities of $\mathrm{UE}_{2}$ when we have different target data rate values. Noting that, the outage probability of $\mathrm{UE}_{2}$ for our proposed scheme and HDU-CNOMA scheme changes as the target data rate changes. In addition, the performance of $P_{\text {out }}^{U E_{2}, D L}$ at $R_{1}=R_{2}=0.5 \mathrm{bit} / \mathrm{s} / \mathrm{Hz}$ is better than the permanence of that when $R_{1}=R_{2}=1 \mathrm{bit} / \mathrm{s} / \mathrm{Hz}$ either in our proposed scheme or in HDU-CNOMA scheme. However, in both cases, the performance of our proposed scheme is still better than the HDL-CNOMA scheme.

The system outage throughput for our proposed scheme and HDU-CNOMA scheme is shown in Fig. 7. We can notice from this figure that the throughput for our proposed scheme is greater 


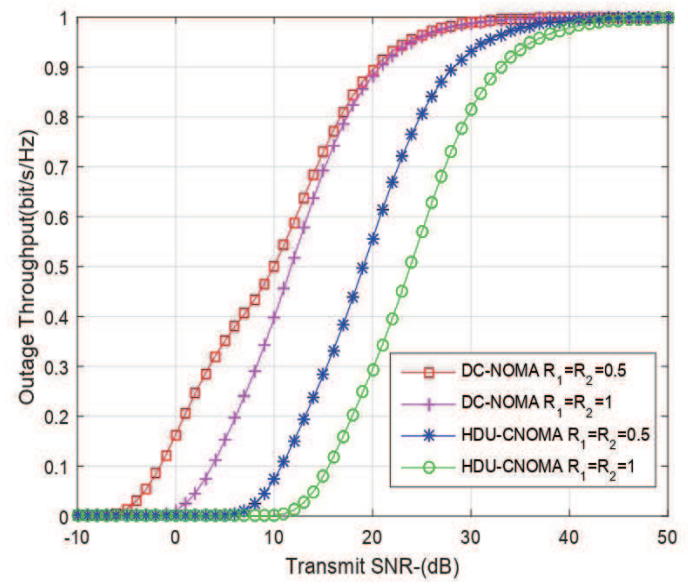

Figure 7: System outage throughput (bits/s/Hz) for DC-NOMA scheme, and HDU-CNOMA scheme at $R_{1}=R_{2}=1 \mathrm{bit} / \mathrm{s} / \mathrm{Hz}$.

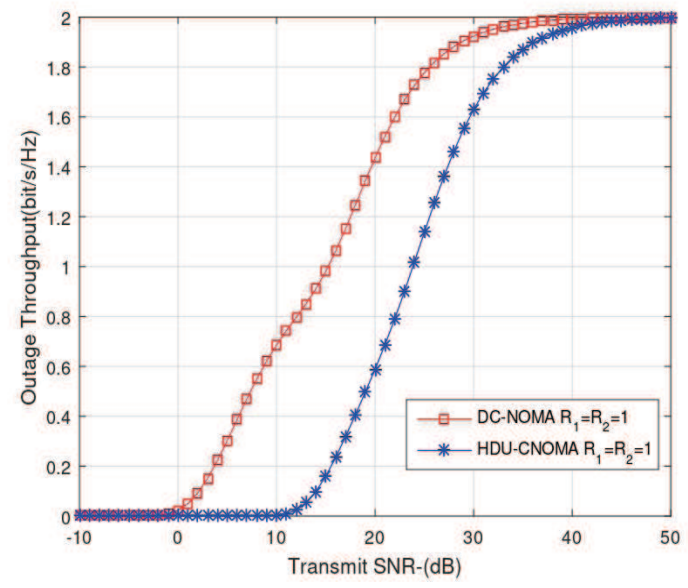

Figure 8: System outage throughput (bits/s/Hz) for DC-NOMA scheme, and HDU-CNOMA scheme at $R_{1}=R_{2}=0.5,1 \mathrm{bit} / \mathrm{s} / \mathrm{Hz}$.

than the HDU-CNOMA scheme. In particular, the performance gains that are achieved by the proposed scheme results from several reasons. First, we can increase the power level for the two users from BS because the power in BS is un-constrained. Second, the cooperation between both users in the cooperative phase assists $\mathrm{UE}_{1}$ to easily decode $s_{2}^{\prime}$, resulting in an increase in the SINR and an improvement in the total performance of the system.

In contrast, HDU-CNOMA has lower outage throughput due to that the HDU-CNOMA loss more power for the interference-free uplink transmission in the cooperative phase,besides, the loss in allocated power for the strong user while it attempts to help the weak user. Its noted that at high SNR, the outage throughput for the proposed scheme is almost to that in the HDU-CNOMA scheme because the outage probabilities for both schemes are almost the same at high SNR. In other words, the outage probabilities equations at high SNR for the proposed scheme is approximated to the outage probabilities equations for HDU-CNOMA scheme which lead to the same throughput in both systems.

Figure 8 illustrates the system outage throughput of our proposed scheme and HDU-CNOMA scheme at different values of target data rates (i.e., $R_{1}=R_{2}=0.5,1 \mathrm{bit} / \mathrm{s} / \mathrm{Hz}$ ). Moreover, when the target data rate values are small, the outage throughput is high. In contrast, when the target data rate values are large, the outage throughput is low. Although we have different values of target data rates, the performance of our scheme is still better than HDU-CNOMA scheme.

\section{CONCLUSION AND FUTURE RESEARCH WORK}

\subsection{Conclusion}

In this paper, we proposed DC-NOMA scheme to increase the spectral efficiency and throughput of cooperative NOMA scheme. We proposed a similar, yet different approach to the other cooperative NOMA scheme. Particularly, the weak user sent a copy of its message to the strong user in order to obtain perfect decoding for the weak user message at the strong user. The transmission occurred in two phases; the downlink phase which is the phase used to transmit the superimposed signal to both users, and the cooperative phase which used to implement the cooperation between the weak and strong users. To evaluate the proposed scheme's performance, we depraved the corresponding outage probability, diversity order, and system outage throughput. The performance of the system was improved in the proposed scheme and this shown by using numerical results. The proposed scheme outperforms the HDU-CNOMA scheme in terms of spectral efficiency. The outage throughput was analyzed in order to study the (QoS) for the link and to evaluate the spectral efficiency. The numerical results for our proposed scheme in-term of the outage throughput demonstrated the better performance over the HDU-CNOMA scheme.

\subsection{Future Research Work}

The main future work for this paper is given as the following; when the weak user $\mathrm{UE}_{2}$ transmits its signal to the strong user $\mathrm{UE}_{1}$, it can simultaneously transmit interference-free uplink superposed 
signal to the BS during the cooperative phase.

Another idea which can be applied is the idea of a relay. In this future work, the relay can act between the BS and the two users to perform decode and forward operation for the signals during the transmission.

\section{REFERENCES}

1. Talwar, S., D. Choudhury, K. Dimou, E. Aryafar, B. Bangerter, and K. Stewart, "Enabling technologies and architectures for 5G wireless," In2014 IEEE MTT-S International Microwave Symposium (IMS2014), 1-4, IEEE, June 1, 2014.

2. Wang, P., Y. Li, L. Song, and B. Vucetic, "Multi-gigabit millimeter wave wireless communications for 5G: From fixed access to cellular networks," IEEE Communications Magazine, Vol. 53, No. 1, 168-178, Jan. 162015.

3. Bharathi, S., L. Nandita, N. Venkateswaran, and R. Subhashini, "Performance analysis of non orthogonal multiple access technique with precoding," In2017 International Conference on Wireless Communications, Signal Processing and Networking (WiSPNET), 1878-1882, IEEE, Mar. 22, 2017.

4. Md Shipon, A., "Non-orthogonal multiple access (NOMA) for cellular wireless communications," 2017.

5. Li, Y., Y. Li, X. Chu, Y. Ye, and H. Zhang, "Performance analysis of relay selection in cooperative NOMA networks," IEEE Communications Letters, Vol. 23, No. 4, 760-3, Feb. 8, 2019.

6. Nasir, A. A., H. D. Tuan, H. H. Nguyen, T. Q. Duong, and H. V. Poor, "Signal superposition in NOMA with proper and improper Gaussian signaling," IEEE Transactions on Communications, Vol. 68, No. 10, 6537-51, July 9, 2020.

7. Saito, Y., Y. Kishiyama, A. Benjebbour, T. Nakamura, A. Li, and K. Higuchi, "Non-orthogonal multiple access (NOMA) for cellular future radio access," In2013 IEEE 77th Vehicular Technology Conference (VTC Spring), 1-5, IEEE, June 2, 2013.

8. Makki, B., K. Chitti, A. Behravan, and M. S. Alouini, "A survey of NOMA: Current status and open research challenges," IEEE Open Journal of the Communications Society, Vol. 1, 179-89, Jan. 28, 2020.

9. Wei, Z., J. Yuan, D. W. Ng, M. Elkashlan, and Z. Ding, "A survey of downlink non-orthogonal multiple access for 5G wireless communication networks," arXiv preprint arXiv:1609.01856, Sep. 7, 2016.

10. Higuchi, K. and A. Benjebbour, "Non-orthogonal multiple access (NOMA) with successive interference cancellation for future radio access," IEICE Transactions on Communications, Vol. 98, No. 3, 403-14, Mar. 1, 2015.

11. Islam, S. R., N. Avazov, O. A. Dobre, and K. S. Kwak, "Power-domain non-orthogonal multiple access (NOMA) in 5G systems: Potentials and challenges," IEEE Communications Surveys \& Tutorials, Vol. 19, No. 2, 721-42, Oct. 25, 2016.

12. Nikopour, H. and H. Baligh, "Sparse code multiple access," In2013 IEEE 24th Annual International Symposium on Personal, Indoor, and Mobile Radio Communications (PIMRC), 332-336, IEEE, Sep. 8, 2013.

13. Sharma, S. and K. Deka, "Sparse code and hybrid multiple access techniques," In5G and Beyond Wireless Systems, 85-105, Springer, Singapore, 2020.

14. Benjebbovu, A., A. Li, Y. Saito, Y. Kishiyama, A. Harada, and T. Nakamura, "Systemlevel performance of downlink NOMA for future LTE enhancements," In2013 IEEE Globecom Workshops (GC Wkshps), 66-70, IEEE, Dec. 9, 2013.

15. Ding, Z., Z. Yang, P. Fan, and H. V. Poor, "On the performance of non-orthogonal multiple access in 5G systems with randomly deployed users," IEEE Signal Processing Letters, Vol. 21, No. 12, 1501-5, Jul. 30, 2014.

16. Timotheou, S. and I. Krikidis, "Fairness for non-orthogonal multiple access in 5G systems," IEEE Signal Processing Letters, Vol. 22, No. 10, 1647-51, Mar. 26, 2015.

17. Laneman, J. N., D. N. Tse, and G. W. Wornell, "Cooperative diversity in wireless networks: Efficient protocols and outage behavior," IEEE Transactions on Information Theory, Vol. 50, No. 12, 3062-80, Nov. 30, 2004.

18. Chen, X., C. Zhong, C. Yuen, and H. H. Chen, "Multi-antenna relay aided wireless physical layer security," IEEE Communications Magazine, Vol. 53, No. 12, 40-6, Dec. 17, 2015. 
19. Zhang, J., L. Dai, Y. Zhang, and Z. Wang, "Unified performance analysis of mixed radio frequency/free-space optical dual-hop transmission systems," Journal of Lightwave Technology, Vol. 33, No. 11, 2286-93, Jun. 1, 2015.

20. Ng, D. W. and R. Schober, "Cross-layer scheduling for OFDMA amplify-and-forward relay networks," IEEE Transactions on Vehicular Technology, Vol. 59, No. 3, 1443-58, Dec. 31, 2009.

21. Ding, Z., M. Peng, and H. V. Poor, "Cooperative non-orthogonal multiple access in 5G systems," IEEE Communications Letters, Vol. 19, No. 8, 1462-5, June 3, 2015.

22. Men, J. and J. Ge, "Non-orthogonal multiple access for multiple-antenna relaying networks," IEEE Communications Letters, Vol. 19, No. 10, 1686-9, Aug. 24, 2015.

23. Zhang, Z., Z. Ma, M. Xiao, Z. Ding, and P. Fan, "Full-duplex device-to-device-aided cooperative nonorthogonal multiple access," IEEE Transactions on Vehicular Technology, Vol. 66, No. 5, 4467-71, Aug. 12, 2016.

24. Kim, J. B. and I. H. Lee, "Non-orthogonal multiple access in coordinated direct and relay transmission," IEEE Communications Letters, Vol. 19, No. 11, 2037-40, Aug. 31, 2015.

25. Zhong, C. and Z. Zhang, "Non-orthogonal multiple access with cooperative full-duplex relaying," IEEE Communications Letters, Vol. 20, No. 12, 2478-81, Sep. 20, 2016.

26. Wei, Z., L. Dai, D. W. Ng, and J. Yuan, "Performance analysis of a hybrid downlink-uplink cooperative NOMA scheme," In2017 IEEE 85th Vehicular Technology Conference (VTC Spring), 1-7, IEEE, Jun. 4, 2017.

27. Belmekki, B. E., A. Hamza, and B. Escrig, "Performance analysis of cooperative NOMA at intersections for vehicular communications in the presence of interference," Ad Hoc Networks, Vol. 98, 102036, Mar. 1, 2020.

28. Yang, W., G. Durisi, T. Koch, and Y. Polyanskiy, "Quasi-static multiple-antenna fading channels at finite blocklength," IEEE Transactions on Information Theory. Vol. 60, No. 7, 4232-65, Apr. 18, 2014.

29. Wei, Z., D. W. Ng, and J. Yuan, "Power-efficient resource allocation for MC-NOMA with statistical channel state information," In2016 IEEE Global Communications Conference (GLOBECOM), 1-7, IEEE, Dec. 4, 2016.

30. Kim, J. B. and I. H. Lee, "Capacity analysis of cooperative relaying systems using nonorthogonal multiple access," IEEE Communications Letters, Vol. 19, No. 11, 1949-52, Aug. $25,2015$.

31. Chen, Z., J. Yuan, and B. Vucetic, "Analysis of transmit antenna selection/maximal-ratio combining in Rayleigh fading channels," IEEE Transactions on Vehicular Technology, Vol. 54, No. 4, 1312-21, Aug. 8, 2005.

32. Xu, L., H. Xing, Y. Deng, A. Nallanathan, and C. Zhuansun, "Fairness-aware throughput maximization for underlaying cognitive NOMA networks," IEEE Systems Journal, June 11, 2020.

33. Chen, Z., J. Yuan, and B. Vucetic, "Analysis of transmit antenna selection/maximal-ratio combining in Rayleigh fading channels," IEEE Transactions on Vehicular Technology, Vol. 54, No. 4, 1312-21, Aug. 8, 2005.

34. Li, X., M. Liu, C. Deng, P. T. Mathiopoulos, Z. Ding, and Y. Liu, "Full-duplex cooperative NOMA relaying systems with I/Q imbalance and imperfect SIC," IEEE Wireless Communications Letters, Vol. 9, No. 1, 17-20, Sep. 4, 2019.

35. Hildebrand, F. B., "Introduction to numerical analysis," Courier Corporation, 1987.

36. Chae, S. and M. Rim, "On the definition of diversity order based on renyi entropy for frequency selective fading channels," Entropy, Vol. 19, No. 4, 179, Apr. 2017.

37. Yazaki, T. and Y. Sanada, "Throughput performance of non-orthogonal multple access with joint detection in far user," In2015 International Symposium on Intelligent Signal Processing and Communication Systems (ISPACS), 537-541, IEEE, Nov. 9, 2015.

38. Chi, K., Z. Chen, K. Zheng, Y. H. Zhu, and J. Liu, "Energy provision minimization in wireless powered communication networks with network throughput demand: TDMA or NOMA," IEEE Transactions on Communications, Vol. 67, No. 9, 6401-14, June 7, 2019.

39. Lavery, R. J., "Throughput optimization for wireless data transmission," MCs Thesis, Polytechnic University, June 2001.

40. Tabassum, H., M. S. Ali, E. Hossain, M. Hossain, and D. I. Kim, "Non-orthogonal multiple access (NOMA) in cellular uplink and downlink: Challenges and enabling techniques," arXiv preprint arXiv:1608.05783, Aug. 20, 2016. 